\title{
Mathematical Model of Gases Flux in Boundary Layer
}

\author{
Valentini $\mathbf{R}^{1}$, Vasenev I.I ${ }^{2, *}$, Nurgaliev I.S ${ }^{3}$ \\ ${ }^{1}$ Full Professor at University of Tuscia,Department of Forest Science and Environment, \\ Via De Lellis, 01100 Viterbo Italy \\ ${ }^{2}$ Head of Ecology Chair, Head of Laboratory Agro-ecological Monitoring, Modeling and Forecasting in Ecosystems, Russian State \\ Agrarian University - Moscow Agricultural Academy named after K.A.Timiryazev \\ ${ }^{3}$ Leading Researcher at the same Laboratory \\ *Corresponding Author: ildus58@mail.ru
}

Copyright (C) 2013 Horizon Research Publishing All rights reserved.

\begin{abstract}
Analytical dynamic model of the turbulent flux in the three-layer boundary system is presented. Turbulence is described as a presence of the non-zero vorticity. The generalized advection-diffusion-reaction equation is derived for an arbitrary number of components in the flux. The fluxes in the layers are objects for matching requirements on the boundaries between the layers. Different types of transport mechanisms are dominant on the different levels of the layers.
\end{abstract}

Keywords Green House Gas, Mathematical Model, Turbulent Flux, Vorticity, Micrometeorology, Material Point, Turbulent Diffusion.

\section{Rationale}

Modeling carbon dioxide and other green house gases (GHG) budgets at the local, regional and global scale is a fundamental task for understanding the current and future behavior of the climate cycles and to work out strategies in the decision making for sustainable development [1]. Mathematical models are effective and often the only available tools and instruments for testing the claims of GHG emission reduction. Building adequate models, especially in the presence of complex phenomena such as turbulence is a big challenge [2]. Further complications may be introduced by the variability of fluxes in mixed landscapes, by the physical aspects in measuring covariance of fluxes and complex atmospheric layer dynamics, as well as the problem of interpretation, gap filling, footprint identification and date aggregation. Therefore, despite the fact that many investigators are producing estimates of annual carbon exchange, it is imperative to clarify and to account for the key limiting factors imposed to the eddy covariance method.
For example, well written just recently published book by Dr. George Burba [3], as well as his previous multiple publications, as an instrumental introduction to the subject and a thorough collection of useful state of the art articles [4] introduce simplified "Eddy Flux" as a product of the averaged air density and correlation term (time-averaged product) between fluctuations of the vertical air velocity and concentration fluctuations of the targeted gas component. The authors carefully perform necessary assessment of different sources of the errors. We try to use new platform to simulate the turbulent model and to account neglected component of the flow, such as vorticity induced turbulent diffusion in gas flux modeling (do not confuse with thermal diffusion: which is in English language literature called molecular diffusion though both types of diffusivity are molecular). Our approach is based on introducing vorticity and a new conception of the material point, constructed and effectively used by one of the authors in cosmology [5-7] and in modeling global social (demographic, economic) and natural (climatic) processes $[15,16]$.

\section{Introduction}

As usual with this type of problems, the system is represented "as simple as possible but not simpler" as the famous saying by A. Einstein calls. Atmosphere, canopy and soil are represented locally by flat horizontal layers, and the dominant transport mechanisms are identified in Figure 1. The fluxes between the layers have to meet the matching conditions required by the theory of partial differential equations, and the development of flux models encounters a series of complex additional requirements at the boarders between media, one of the difficulties being the incomplete knowledge of the physical laws of the turbulence. 


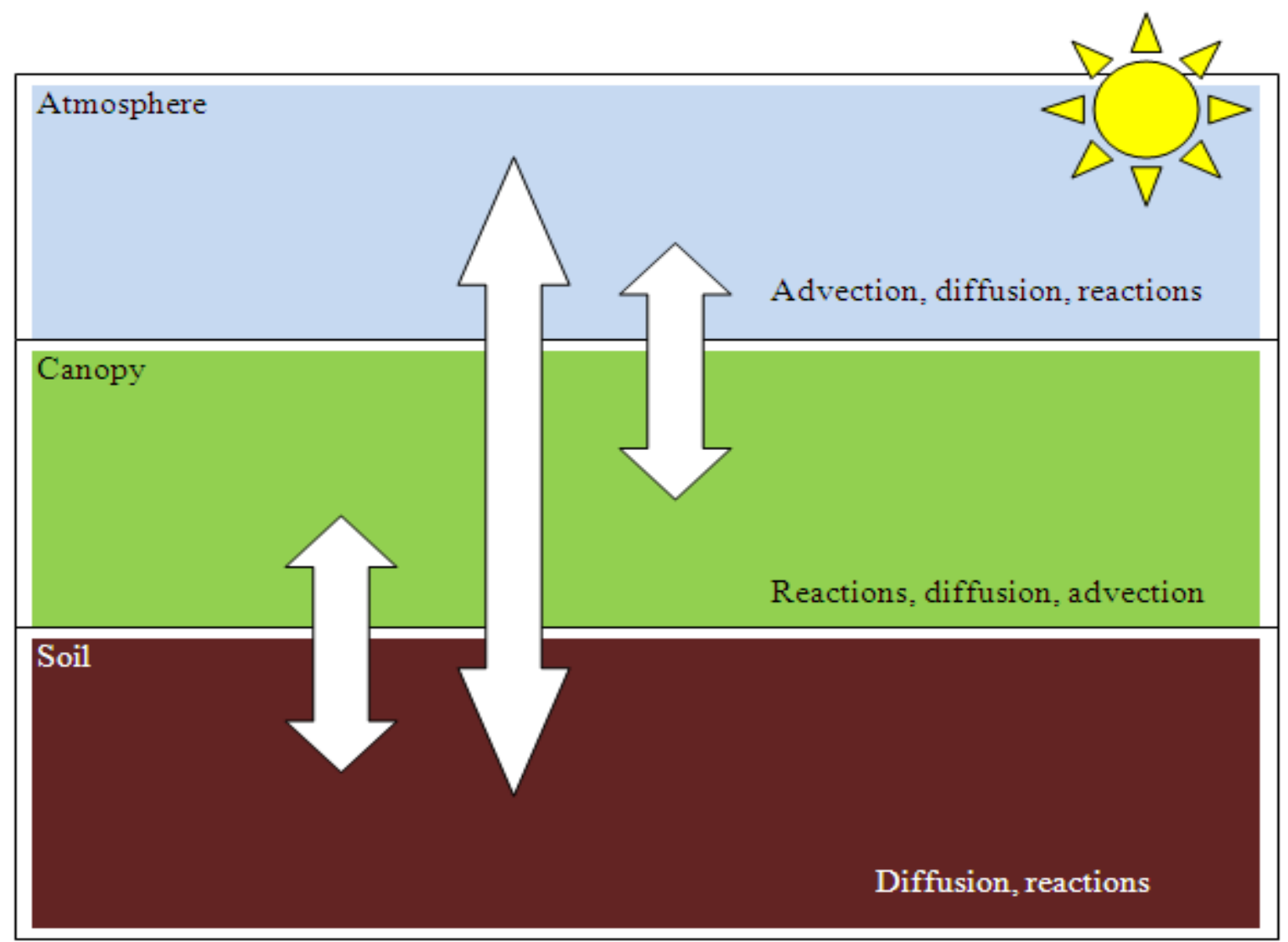

Figure 1. The components of the model.

This problem will be approached in the present work by using the vorticity concept. The eddy covariance method, in its currently accepted form, serves as a compromise available at the moment for addressing the multiple questions still open with regard to the turbulent flow measurement and monitoring. In the main eddy covariance expression, two diffusive fluxes have been neglected so far: the molecular diffusion and the turbulent diffusion. The first is much less than the second, and can be neglected indeed. But the second is key factor in the model and have to be accounted. As some authors do (see bibliography in [3] and in [4]), we forced to limit ourselves to the simple enough developed turbulence mechanism which covers the important aspects of the gas transport in the boundary layer. But, first of all, diffusive transport is main input/output mechanism of gases and, therefore, has to be counted and estimated consciously in the layer flux balance. And, secondly, if we plan to build self-consistent models of the gas circulation within all three subsystems, diffusion is the main phenomenon in the soil layer. Therefore, in the expression of eddy covariance flux, accounting speed of air has to be combined by the speed of the considered gas relative to "air", i.e. all other ones taken together as a sort of system of reference. That will be building model as simple as we can, but ignoring coexistence of the turbulent diffusion transport among the others would be oversimplification.
Therefore the problem arises of instrumental discrimination between speed of air and, specifically target gas say, carbon dioxide along with mathematical modeling. Other constraints of the method can be found in [8-11].

\section{New Concept of Material Point}

In the traditional approach of the eddy covariance method, one of the basic concepts is the fluid parcel, defined in Wikipedia as 'a very small amount of fluid, identifiable throughout its dynamic history while moving with the flow' referring to [17].

The new approach to the turbulent flow model that will be dealt with in this article starts by reconsidering the basic concept of the 'material point' itself displacing parcel concept. The usual meaning of a small, dimensionless body carrying a given mass will be called material point of type 1 . The new concept that we introduce in this article, is that of material point of type 2 . While the type 1 point is characterized by mass, this type 2 material point is characterized by its density, not mass. Its motion has attributes of continuous media, i.e. deformations and torsions.

Hereby we get rid of to the old sophism "let us call material particle material point and will treat it as a point." 
Sorry, material point is material. Therefore it has inalienable ability to be oriented relative to other material objects. And calling it point should not eliminate this essential ability. Treating material point, just because its name, as it was geometrical point is sophism. Description of material point has to include his degrees of freedom, specifically, rotational and distortional ones, characteristic to anything material.

This revelation brings us to reconsideration of the flux. A new aspect of the new model is awareness on necessity of projecting velocity vector $V_{\alpha}$ to configuration space of radius-vectors $\mathrm{R}^{\beta}$ to manipulate with velocities' Cartesian coordinates:

$$
V_{\alpha}=\mathrm{H}_{\alpha \beta} \mathrm{R}^{\beta},
$$

because velocity vector is an object of the different (tangential) space. For the fundamental geometrical aspects of this statement see [12].

Here in (1) $\mathrm{H}_{\alpha \beta}$ is advection-distortion-vortex tensor (affinor, in old terminology, as it is called in [12], representation of an projection operator), $\mathrm{R}^{\beta}$ is radius-vector of the material point. Splitting tensor $H_{\alpha \beta}$ into three parts, responsible for expansion $\theta \delta_{\alpha \beta} / 3$, shear $\sigma_{\alpha \beta}$ and vorticity $\omega_{\alpha \beta}$ is a novel tool to describe turbulence in the boundary layer agro-meteorology, accounting new degrees of freedom of the material particle as the material point of the second type:

$$
H_{\alpha \beta}=\theta \delta_{\alpha \beta} / 3+\sigma_{\alpha \beta}-\omega_{\alpha \beta} .
$$

\section{GHG Flux Model Equations}

Hydrodynamic Euler equations split into system (3) of 3 sets of equations

$$
\begin{aligned}
\frac{d \vartheta}{d t}+\frac{1}{3} \theta^{2}+\sigma^{2}-\omega^{2}=-\boldsymbol{F}_{\alpha \alpha} \\
\sigma^{2}=\sigma_{\alpha \beta} \sigma_{\alpha \beta}, \omega^{2}=\omega_{\alpha \beta} \omega_{\alpha \beta}, \frac{d \rho}{d t}=-\rho \vartheta, \quad \text { (3) } \\
\frac{d \sigma_{\alpha \beta}}{d t}+\frac{2}{3} \vartheta \sigma_{\alpha \beta}+\sigma_{\alpha \gamma} \sigma_{\gamma \beta}-\frac{1}{3} \delta_{\alpha \delta} \sigma^{2} \\
+\omega_{\alpha \gamma} \omega_{\gamma \beta}+\frac{1}{3} \delta_{\alpha \delta} \omega^{2}=-F_{(\alpha \beta)} \\
+\frac{1}{3} \delta_{\alpha \delta} F_{\gamma \gamma} \\
\frac{d \omega_{\alpha \beta}}{d t}+\frac{2}{3} \vartheta \omega_{\alpha \beta}+\sigma_{\alpha \gamma} \omega_{\gamma \beta}+\sigma_{\alpha \gamma} \omega_{\gamma \beta}+\omega_{\alpha \gamma} \delta_{\gamma \beta}=-F_{[\alpha \beta]} .
\end{aligned}
$$

Here $F_{\alpha \beta}$ is a tensor of the external forces gradient. Dynamics of the turbulent flux (3) is much more complex than of laminar flux and flux measurement methods need including turbulence-related terms.

\section{Advection-Diffusion-Reaction Equations}

For a more general description structure (e.g. eddies) formation we get equation of the multi-component reaction-diffusion type

$$
\frac{\partial x_{i}}{\partial t}=f_{i}\left(\left\{x_{j}\right\}\right)+\nabla_{k} D_{j}^{k}(r) \nabla^{j} x_{i}
$$

Substances diffusion and transfer of thermal energy are described by the same class equations. This equation is extremely universal and can be applied in modeling the broad range of processes taking place in agroindustry and in its energetics. The most impressive new application is a thermochemical decomposition of organic material such us agro manure at elevated temperatures without the participation of oxygen (pyrolysis) and production of designed fuels. It goes without saying that migration and generating (reacting) of gases in soil described by this equation.

The geometries of the subsystems are also considered in the modeling of the growth kinetics as a crucial factor. New class of equations called advection-diffusion-reaction (http://arxiv.org/pdf/1210.4091v2.pdf) was derived as following

$$
\frac{\partial \rho_{i}}{\partial t}=-\frac{1}{3} H_{i} \rho_{i}+f\left(\left\{\rho_{i}\right\}\right)+\nabla\left[D_{i}\left(\left\{\rho_{i}\right\}\right) \nabla \rho_{i}\right]
$$

This equation describes arbitrary amount of material components with densities $\rho_{i}$, parameters $H_{i}$ - diagonal elements of matrix in (1) - are responsible for an advection change of density, and coefficients of the effective diffusion $D_{i}$, generalized and adopted when needed. It may also provide nonlinear evolution scenarios for evolution of the multi-component reacting media in the different systems such as agro-bio-geo fluxes. The nonlinear term $f\left(\left\{\rho_{i}\right\}\right)$ stands for reactions between the components. The fluxes in the layers are objects for matching requirements on the boundaries between the layers. These requirements are fulfilled by appropriate identification of the constants of integration.

One of the new features of the nonlinear dynamic processes described by given equation is the existence of the so called threshold effects. This means that we may expect emergence and ability to long existence of some eddies and grow some of them to scales intensities of tornado before getting destroyed up.

\section{Speed of Sound in Turbulent Flux}

Next aspect in using physical laws in the presence of turbulence is taking into account vorticity in Doppler Effect for measurement of speed of flux. Traditionally eddy covariance theory refers to standard Doppler Effect whereas 
it is based on consideration of the flux as turbulent. In self-consistent model the basic equation describing (ultra)sound needed to be one for turbulent flux, e.g. Blokhintsev- Howe equation [13, 14]. In the adiabatic approximation the equation holds

$$
\begin{gathered}
\left\{\frac{D}{D t}\left(\frac{1}{c^{2}} \frac{D}{D t}\right)+\frac{1}{c^{2}} \frac{D v}{D t} \nabla-\nabla^{2}\right\} B=\operatorname{div} L-\frac{1}{c^{2}} \frac{D v}{D t} L, \\
B=H+v^{2} / 2, \frac{D}{D t}=\frac{\partial}{\partial t}+v \nabla, L=\Omega \times v-T \nabla S
\end{gathered}
$$

Here $H$ is enthalpy, $\mathbf{\Omega}=\boldsymbol{r o t} v$ is vorticity, $S$ - entropy, $T$ temperature, $v$ is flux speed, $c$ is sound speed, $t$ is time. Note that drag enthalpy $B$ is connected to sound pressure $p$ as $\partial p / \partial t=\rho D B / D t$ ( $\rho$ is mass density of the media). Blokhintsev- Howe equation is derived as a consequence of impulse and mass conservation as well as equation of the state of the ideal gas. LHS of it correspond to the transfer of the sound in the arbitrary non-homogeneous flux and RHS characterizes the sources of the sound connected to character of the flux such as presence of vorticity and entropy gradient. As one can see, sound equation much more complicated than those traditionally used for deriving Doppler Effect in the frame of eddy covariance method.

\section{Conclusions}

"The dynamics of the turbulent flow of GHGs on the boundary layer, as the present mathematical model demonstrates, is much more complicated compared to the vortex free motion. Therefore, the eddy covariance method, as an existing broadly accepted and successfully working instrument, can be further developed towards more detailed models that account for vorticity. Therefore, we expect that the existing unsolved problems in the boundary flux model, such as energy balance, the difference in tower-based monitoring and camera based methods, can be tackled by using the models that consider the vortex flow

\section{Acknowledgements}

This research was funded by Government of Russian Federation, Grant № 11.G34.31.0079.

\section{REFERENCES}

[1] Vasenev I.I., Valentini R. Experience in Organization Green House Gases Monitoring, C- and N-stockpiles in the Anthropologically Changed Ecosystems in the European Part of Russia. Report on the 1st Annual Scientific-Organizational Conference, Laboratory for Agro-ecological Monitoring, Modeling and Forecasting in Ecosystems (LAMP), Russian State Agrarian University Moscow Agricultural Academy named after K.A.Timiryazev.
December 12, 2012.

[2] Landau L.D., Lifshitz E.M. (1987). Fluid Mechanics. Vol. 6 (2nd ed.). Butterworth-Heinemann. 1959

[3] Burba G. Eddy Covariance Method for Scientific, Industrial, Agricultural and Regulatory Applications. A Field Book on Measuring Ecosystem Gas Exchange and Areal Emission Rates. ISBN 978-0-615-76827-4 . LI-COR Biosciences, Lincoln, Nebraska, 332 pp.

[4] Aubinet, M., Vesala T., and Papale D. (Eds.), 2012. Eddy Covariance: A Practical Guide to Measurement and Data Analysis. Springer, Dordrecht, London, New York, 442 pp.

[5] Nurgaliev I.S. «Singularities Are Averted by Vortices». Gravitation and Cosmology, 2010, Vol. 16, No. 4, pp. 313-315.

[6] Nurgaliev I.S.. Modern astronomical knowledge as component of general education for sustainable development. Astronomical and Astrophysical Transactions (AApTr), 2012, Vol. 27, Issue 3, pp. 429-430. ISSN 1055-6796, Cambridge Scientific Publishers.

[7] Nurgaliev I.S. "Confirmation of Cosmological Bounces Predicted by Alexander Friedmann." International Journal of. Modern Physics: Conference Series, Vol. 3 (2011), pp 281-285.

[8] Baldocchi D.D. "Assessing the eddy covariance technique for evaluating carbon dioxide exchange rates of ecosystems: past, present and future. Global Change Biology, Volume 9, Issue 4, pages 479-492, April 2003.

[9] .Webb, E. K., Pearman, G. I., and Leuning, R.: 1980, 'Correction of Flux Measurements for Density Effects Due to Heat and Water Vapour Transfer', Quart. J. Roy. Meteorol. Soc. 106, 85-100.

[10] Wyngaard J.C. 1990: Scalar fluxes in the planetary boundary layer -- Theory, modeling, Boundary-Layer Meteorology and measurement. Boundary Layer Meteorology, 50, 49-75.

[11] Fuehrer P. L., C. A. Friehe. Flux Corrections Revisited. Boundary-Layer Meteorology, 102: 415-457, 2002.

[12] Rashevsky P.K. Riemann Geometry and Tensor Analysis (in Russian), Nauka. Eds, Moscow, 1967.

[13] Blokhintsev D.I., Acoustics of a Non-homogeneous Moving Medium, Gostekhizdat, 1945 (English translation: N.A.C.A. Technical Memorandum no. 1399 (1956)).

[14] Howe D.I. Acoustics of Fluid-Structure Interactions, Cambridge University Press, Cambridge, 1998.

[15] Nurgaliev I.S. Physical Kinetics of Demography. Economic Strategies. - 2009. - N 1. - pp. 170-175. www.intelros.ru/pdf/ps/02/31.pdf

[16] Nurgaliev I.S. Turbulency of the New Risks Demand Revising Strategies of Development. Economic Strategies 2011, $\quad \mathrm{N} \quad 6, \quad$ pp. $56-60$ http://www.inesnet.ru/magazine/mag_archive/?mid=105\&ci $\mathrm{d}=2416$ \#article 2416

[17] Batchelor, George K.(1973). An introduction to fluid dynamics. Cambridge: Cambridge University Press. ISBN 0-521-09817-3 